\title{
Free energy surfaces of ionic adsorption in cholesterol-free and cholesterol-rich phospholipid membranes
}

\author{
Jordi Martí \\ Department of Physics, Technical University of Catalonia-Barcelona Tech, B5-209 Northern Campus \\ UPC. Jordi Girona, 1-3. 08034 Barcelona. Catalonia. Spain.
}

(Received 00 Month 20XX; final version received 00 Month 20XX)

\begin{abstract}
Free energy surfaces associated to the adsorption of metal cations $\left(\mathrm{Na}^{+}, \mathrm{K}^{+}, \mathrm{Ca}^{2+}, \mathrm{and}^{\mathrm{Mg}}{ }^{2+}\right)$ in biological environments have been computed by metadynamics simulations. In all cases, the systems were modeled using the CHARMM36 force field. The free-energy landscapes unveil specific binding behavior of metal cations. So, $\mathrm{Na}^{+}$and $\mathrm{K}^{+}$are more likely to stay in the aqueous solution, and can easily bind to a few lipid oxygens by overcoming low free-energy barriers. Differently, $\mathrm{Ca}^{2+}$ is most stable when bound to four lipid oxygens of the membranes, rather than being hydrated in the aqueous solution. Finally, $\mathrm{Mg}^{2+}$ is tightly hydrated, and can hardly lose a hydration water and bind directly to the membranes. When cholesterol is included inside the membrane at concentration up to 50\%, the resulting free energy landscapes reveal the competition between binding of sodium to water and to lipid head groups, although the binding competitiveness of lipid head groups is diminished by cholesterol contents. When cholesterol concentration is greater than $30 \%$, the ionic binding is significantly reduced, which coincides with the phase transition point of DMPC-cholesterol membranes from a liquid-disordered phase to a liquid-ordered phase.
\end{abstract}

Keywords: Free energy, phospholipid membrane, metadynamics, cholesterol.

\section{Introduction}

Ion adsorption on biological systems has drawn great attention during last decades[1]. Among an increasing variety of ion species it has been observed that, when ion-water interactions are dominant, specific cation effects are of particular importance[2]. In the case of biomembranes in physiological environments, the interactions between metal cations and charged lipid headgroups are essential for the regulation of membrane properties and membrane functioning. These regulations deeply depend on the ion specificity[3, 4]. A number of experiments suggested that metal cations are normally bound to the phosphate and carbonyl regions of phospholipid membranes [5-7].

The binding of metal cations at membranes in an aqueous medium can result in several possible bound configurations, as a consequence of the loss of water molecules and the gain of lipid atoms in the ion's first coordination shell[8, 9]. Binding rates and Gibbs free-energies of metal cations can be estimated from experiments[8]. Nevertheless most experiments and simulation works on ionic binding to phospholipid membranes have been performed in cholesterol-free environments. Cholesterol is a crucial component in mammalian cell membranes, constituting up to $50 \%$ of their weight[10]. It can modulate the structural and mechanical properties of the membrane and it can induce phase transitions from liquid disordered to liquid ordered phases[11-13]. Therefore, the study of the binding of metal cations to cholesterol-containing membranes is of interest to understand ionic binding in realistic cellular environments. Experiments by Iraolagoitia et al. showed that cholesterol signif- 
icantly reduced the $\mathrm{Ca}^{2+}$ binding to membranes[14]. More recently, Magarkar et al.[15] performed both experiments and simulations, revealing that increasing cholesterol concentration decreased $\mathrm{Na}^{+}$binding.

Molecular Dynamics (MD) simulations have been extensively employed to investigate the role of metal cations in solvated phospholipid membranes[7, 9, 16-19]. However, the computational study of ion binding to membranes is a very demanding task due to the need of extremely long simulations, required to model ion-membrane binding events. Furthermore, bound configurations are usually separated by high free-energy barriers, making it difficult for MD simulations to sample them adequately[20]. In order to overcome these difficulties, free-energy calculations using enhanced sampling tools have been developed[21]. However, due to the complexity of membrane environments the determination of the proper collective variables, which play a central role in most sampling techniques, is a difficult challenge[22].

In the present paper we have performed a systematic free-energy calculation of binding states of metal cations at phospholipid membrane surfaces using the well-tempered metadynamics methodology[23]. To do this, we previously needed to generate several membrane-cholesterol setups in electrolyte solutions. For these we employed a recent parameterization of the CHARMM force field (CHARMM36) which has been proved to deliver excellent agreement with a significant number of most relevant properties of biomembranes[24]. This fact is crucial in the outcome of the results presented in this paper, which are of course strongly dependent of the particular force field employed (see Catte et al. for a detailed discussion[25]). In addition, we have obtained free energy landscapes of $\mathrm{Na}^{+}$bound to phospholipid membranes of several cholesterol concentrations at infinite ionic dilution. The specific interactions of biologically relevant cations $\mathrm{Na}^{+}, \mathrm{K}^{+}, \mathrm{Ca}^{2+}$, and $\mathrm{Mg}^{2+}$ with cholesterol-free membranes are revealed and interpreted from a free-energy perspective. Further, the competition between ion-water and ion-membrane binding is also studied. Finally, the application of the same methodology to membrane interfaces with higher complexity, namely those including cholesterol effects on $\mathrm{Na}^{+}$binding at phospholipid membranes, will enforce the reliability of the techniques introduced in the present work.

\section{Computational Methods}

One of the hardest challenges in metadynamics simulations is the determination of the most suitable collective variables $(\mathrm{CV})$ to study a given process. In order to obtain the free energy profiles for the partition of a biomembrane by a solute, the normal direction to the plane of the membrane, $Z$, has been typically adopted as the reaction coordinate[26, 27]. Same procedure was adopted for the study of ionic permeation[28, 29], although the problem of ion binding to membranes is more complex, since membranes are made by a large number of lipids with a large number of conformations, with each lipid having several binding sites (usually in headgroups), making it a challenge to define proper and reliable CVs able to describe the interaction between lipids and ions. Moreover, ions are normally hydrated by water molecules, carrying a local solvation shell, in such a way that hydration levels depend on the particular positions of the ions at the membrane surfaces. A significant number of experiments [5-7, 30] and simulation works [7, 9, 16-19, 31, 32] have shown that metal cations directly coordinate with the oxygen atoms of phosphate $\left(\mathrm{PO}_{4}^{-}\right)$and carbonyl $(\mathrm{C}=\mathrm{O})$ groups of lipid molecules, indicating that the oxygens in the lipid headgroup are the binding sites for metal cations. Accordingly, in this work we defined two CVs to describe the ion-membrane binding: the coordination number between a metal ion and lipid oxygens (CLP), and the coordination number between a metal ion and water oxygens (CWT). In the present paper, well-tempered metadynamics[23], an extension of the original metadynamics method[33] has been employed to calculate the free-energy landscape of ion binding to the membrane.

Before performing well-tempered metadynamics simulations, each membrane system was created and fully equilibrated. CHARMM-GUI[34, 35] was employed to generate four sets of lipid bilayer 
systems. Each system consisted of 50 dimyristoylphosphatidylcholine (DMPC) lipid molecules[36, 37], 2500 TIP3P-modified water molecules[38, 39], a metal cation, and one or two $\mathrm{Cl}^{-}$anions to neutralize the system. Each membrane system was equilibrated with MD simulations of length 100 ns in the NPT ensemble at $1 \mathrm{~atm}$ and $303 \mathrm{~K}$. MD simulations for equilibration were performed using NAMD 2.9[40] and the CHARMM36 force field[41]. A time step of 2 fs was used. Covalent bonds with hydrogen atoms of lipids were kept rigid using SHAKE[42], and water molecules were kept rigid using SETTLE[43]. The particle mesh Ewald method was employed to compute long-range electrostatic interactions [44]. The cutoff for Lennard-Jones interactions was set to $12 \AA$, with a switching distance of $10 \AA$ A. Pressure was controlled by the Langevin piston Nosé-Hoover method and the ratio of the unit cell in the $\mathrm{x}-\mathrm{y}$ plane was kept constant[45]. Temperature was controlled by the Langevin dynamics with a damping coefficient of $1 \mathrm{ps}^{-1}[46]$. Areas per lipid were about 60 $\AA^{2}$.

After equilibration and for the first part of the paper, four sets of two-dimensional (2D) welltempered metadynamics simulations were performed in the NVT ensemble in order to calculate the free-energy surfaces of $\mathrm{Na}^{+}, \mathrm{K}^{+}, \mathrm{Ca}^{2+}$, and $\mathrm{Mg}^{2+}$ ions at neutral zwitterionic phospholipid membranes, with no presence of cholesterol. We used NAMD 2.9 together with PLUMED 2 plugin[47] and considered the two CVs described above (CLP, CWT). The coordination number [48] is defined by

$$
s=\sum_{i \in G_{1}} \sum_{j \in G_{2}} s_{i j}
$$

where $s_{i j}$ is a differentiable switching function defined as

$$
s_{i j}=\frac{1-\left(\frac{\left|\mathbf{r}_{i}-\mathbf{r}_{j}\right|-d_{0}}{r_{0}}\right)^{6}}{1-\left(\frac{\left|\mathbf{r}_{i}-\mathbf{r}_{j}\right|-d_{0}}{r_{0}}\right)^{12}} .
$$

For CLP, $G_{1}$ is the metal ion, $G_{2}$ is all the lipid oxygens. For CWT, $G_{1}$ is the metal ion, $G_{2}$ is all the water oxygens. The values of $d_{0}$ and $r_{0}$ were determined from the radial distribution function $\mathrm{g}(\mathrm{r})$, which was calculated from unbiased simulations on membrane systems containing 128 DMPC lipids, 6400 water molecules, 46 metal ions, and 46 or $92 \mathrm{Cl}^{-}$ions (corresponding to the ionic concentration of $0.4 \mathrm{M}$ ). All simulations were run for $100 \mathrm{~ns}$. $d_{0}$ is the position of the first peak of $\mathrm{g}(\mathrm{r})$, and $r_{0}$ is the width at half maximum of the peak. The determination of the parameters for coordination number, i.e. $d_{0}$ and $r_{0}$, is essential for the accuracy of the resulting free-energy surfaces. The values for $d_{0}, r_{0}$ and other parameters are listed in Table 1 for the case of cholesterol-free membranes.

For each system long well-tempered metadynamics simulations were performed in the NVT ensemble at the temperature of $303 \mathrm{~K}$ for $\mathrm{Na}^{+}, \mathrm{K}^{+}$and $\mathrm{Ca}^{2+}$. In the case of $\mathrm{Mg}^{2+}$, the simulations were short (100 ns) due to the impossibility to get the ion adsorbed inside the membrane. In the simulations $800 \mathrm{~ns}$ long, each ion was conducted from outside the membrane towards the adsorbed state by metadynamics' biasing and during the process lipid DMPC chains and surrounding water molecules have evolved dynamically to solvate the ions; then coordination numbers have been computed and revealed fluctuations between values of 0 and 8. The Gaussian widths for both CLP and CWT were set to 0.2 . The initial Gaussian deposition rate was $0.3 \mathrm{kcal} / \mathrm{mol}$ per ps, with a bias factor of 5. Despite the NPT ensemble is preferred in simulations of phospholipid membranes, we employed the NVT ensemble in applying well-tempered metadynamics due to technical reasons[47]. However, the 100ns NPT equilibration run brings the membrane to its equilibrium area per lipid, which is then used in the production run to minimize the possible artifacts caused by the application 
of the NVT ensemble. In Figure 1 we show the fluctuations recorded in the values of the collective variables for each class of ion.

For the second part of the paper, we employed six sets of simulations to study the binding of $\mathrm{Na}^{+}$ to neutral zwitterionic phospholipid membranes with different cholesterol content. In this case, all simulations were run for $1000 \mathrm{~ns}$. The technical characteristics of the metadynamics simulations are the same as those reported in Table 1 for sodium. CHARMM-GUI was again employed to generate the six sets of lipid bilayers with cholesterol concentrations [CHOL] varying between $0 \%$ and 50\%. Each system consisted of 72 cholesterol / DMPC molecules, 3600 water molecules and a pair of $\mathrm{Na}^{+}$and $\mathrm{Cl}^{-}$described by the same force fields as in the first part. In contrast to other force fields (such as GROMOS), the CHARMM36 force field generates a less pronounced adsorption of $\mathrm{Na}^{+}$ions on phosphatidylcholine (PC) membranes, which was shown to be consistent with structural X-ray measurements and experiments on chain ordering of PC lipid bilayers in the presence of $\mathrm{NaCl}[24]$. To achieve such agreement with experiment, in CHARMM36 the LennardJones parameters characterizing the interaction of $\mathrm{Na}^{+}$with selected groups in the phospholipid are not calculated following the standard arithmetic combining rules, but they are determined specifically for each atom pair[49]. The need for a specific description of certain pair interactions shows the limitation of point charge force fields, which cannot account for charge transfer or polarization effects [50].

The molar ratios of cholesterol/DMPC were set to $0 / 72(0 \%), 8 / 64$ (11.1\%), 14/58 (19.4\%), 22/50 $(30.6 \%), 30 / 42(41.7 \%)$, and $36 / 36(50 \%)$. Due to the limited size of the system, our simulations can only capture the effect of short wave undulations on the adsorption of ions to the membrane. However, we believe that, being ion-binding presumably a local effect with characteristic length scales in the order of the nanometer, the relevant undulations are the short wavelength modes, which are well accounted for even by small systems[51]. We have investigated the effect of the limited size of the simulated system by comparing the occupancy distribution of the lower ion binding states obtained from unbiased simulations of systems containing 72 and $128 \mathrm{DMPC} /$ cholesterol molecules with $0 \%, 20 \%$, and $30 \%$ of cholesterol. We obtained very similar occupancy distributions for the lower free energy states sampled by unbiased simulations, discarding a large size effect of our simulated system. In these cases, areas per lipid decreased up to about $50 \AA^{2}$, in good agreement with other authors[12].

\section{Free energy surfaces}

\subsection{Part I: Cholesterol-free}

The set of 2D free-energy surfaces (FES) of metal cations bound to DMPC membranes with 0\% of cholesterol content are shown in Figure 2. At the FES of $\mathrm{Na}^{+}$of Figure 2a a staircase pattern can be observed. The $\mathrm{Na}^{+}$ion is in the aqueous solution at CLP $=0$, where it can be hydrated by at most 8 water molecules. When fully dehydrated $(\mathrm{CWT}=0), \mathrm{Na}^{+}$can be bound to at most 7 lipid oxygens. Between these two extreme cases, $\mathrm{Na}^{+}$is bound to oxygens of both water and lipids. The fact that the states at local free energy minima are those with integer numbers of CLP and CWT fully validates our choice of CVs to explore the binding processes, so that the pattern shown in Figure 2a is a consequence of the competition between the binding of the ion to lipids and to water. Indeed, paths of approximately the same binding free energy are found for integer values of CLP and CWT with the same total coordination number (CLP+CWT). The global minimum of the FES is at the $[\mathrm{CWT}=5, \mathrm{CLP}=0]$ state, revealing that in the aqueous solution the most favorable state for $\mathrm{Na}^{+}$is to be hydrated by 5 water molecules. When $\mathrm{Na}^{+}$is bound to the membrane, the stable bound states are located in the region with CLP $\in\{1,4\}$ and $\mathrm{CWT} \in\{1,5\}$ with the total coordination number $(\mathrm{CLP}+\mathrm{CWT}) \in\{4,6\}$. A representative snapshot of a bound state of $\mathrm{Na}^{+}$coordinated with 6 oxygens is shown in Figure 3a. Similar patterns are also present 
in the FES of $\mathrm{K}^{+}$in Figure $2 \mathrm{~b}$, revealing similar binding behaviors of $\mathrm{K}^{+}$and $\mathrm{Na}^{+}$at aqueous membrane interfaces. Because of a larger atomic size, $\mathrm{K}^{+}$has a larger first coordination shell but a relatively lower surface charge density. The global minima states are at $[5,0]$ and $[6,0]$. Therefore, the most favorable configuration for $\mathrm{K}^{+}$in aqueous solution is to be hydrated by $5 \sim 6$ water molecules. When bound to membrane surfaces, $\mathrm{K}^{+}$can be coordinated to more water oxygens and lipid oxygens than $\mathrm{Na}^{+}$. The stable bound states for $\mathrm{K}^{+}$are in the region with $\mathrm{CLP} \in\{1,4\}$ and $\mathrm{CWT} \in\{2,6\}$ with the total coordination number $(\mathrm{CLP}+\mathrm{CWT}) \in\{5,7\}$. We have observed that for monovalent cations $\left(\mathrm{Na}^{+}, \mathrm{K}^{+}\right)$the preferred sites for lipid binding are the oxygens of the carbonyl groups.

The analysis of divalent cations revealed significantly different features. So, we showed above that staying in the aqueous solution is most favorable for the monovalent metal cations $\left(\mathrm{Na}^{+}\right.$, $\left.\mathrm{K}^{+}\right)$. Now we will see that being bound to the membrane surfaces is most stable for $\mathrm{Ca}^{2+}$. The global free-energy minimum at $[2,4]$ in Figure 2c indicates that $\mathrm{Ca}^{2+}$ is preferably bound to 4 lipid oxygens (see Figure $3 \mathrm{~b}$ ), rather than being hydrated in the aqueous solution. In contrast to the stable bound states of $\mathrm{Na}^{+}$and $\mathrm{K}^{+}$with $\mathrm{CLP} \in\{1,4\}$, those for $\mathrm{Ca}^{2+}$ are located in the region with $\mathrm{CLP} \in\{2,6\}$, which suggests a significantly greater affinity of $\mathrm{Ca}^{2+}$ to lipid oxygens than that of monovalent metal cations. The preferred sites for $\mathrm{Ca}^{2+}$ to lipid binding are the oxygens of the phosphate groups. The case of $\mathrm{Mg}^{2+}$ is different from the other ions considered. The bound states of $\mathrm{Mg}^{2+}$ to lipid oxygens $(\mathrm{CLP} \geq 1)$ are separated from the unbound states in the aqueous solution $(\mathrm{CLP}=0)$ by high free-energy barriers, and could not be appropriately sampled by our calculations. This is attributed to the high energy required for partial dehydration of the first hydration shell of $\mathrm{Mg}^{2+}$, which is shown in Figure 4d, where only the case with CLP $=0$ is considered. The strong binding of $\mathrm{Mg}^{2+}$ to its hydration layer has been also reported recently by Allnér et al. They calculated the potential of mean force between $\mathrm{Mg}^{2+}$ and water oxygens and that between $\mathrm{Mg}^{2+}$ and phosphate oxygens, and showed extremely high free-energy barriers for water dehydration and direct phosphate binding[52].

A quantitative representation of the above FES is given in Figure 4, where the free-energies are plotted as a function of CWT at various CLP, and in Table 2, where the most relevant binding states are selected. As shown in Figure 4a, several hydration free-energy basins have been found for CLP = 0 , where $\mathrm{Na}^{+}$is unbound to membranes. The most stable hydration state for $\mathrm{Na}^{+}$is when solvated by 5 water molecules, in agreement with the hydration number measured by experiments[53, 54]. The ion is considered bound to the membrane when CLP $>0$. For $\mathrm{Na}^{+}$at low binding, i.e. for CLP $=1,2,3$, the corresponding minimum free-energies are located at hydration levels CWT $=4,3,2$, respectively, being 5 the total coordination number, which is the same as experimental hydration number[53, 54]. At high binding, i.e. for CLP $=4,5,6$, the most stable hydration levels are CWT $=$ $2,1,0$, respectively, being 6 the total coordination number. The states with CLP $=1,2,3$ and total coordination number of $5 \sim 6$ are the lowest free-energy bound states, which are $1 \sim 2 \mathrm{kcal} / \mathrm{mol}$ higher than the global minimum at $\mathrm{CLP}=0$. A quantitative characterization of the free-energies of those states is given in Table 2. Consequently, those are the most likely bound states of $\mathrm{Na}^{+}$ that one is expected to find in a DMPC membrane immersed in a $\mathrm{NaCl}$ aqueous solution. As it is shown in Figure 4b, the profiles for $\mathrm{K}^{+}$are similar to those for $\mathrm{Na}^{+}$. The hydration with 5 or 6 water molecules is most stable for $\mathrm{K}^{+}$at $\mathrm{CLP}=0$, in agreement with the experimental hydration number $[53,54]$. Free-energy barriers constraining $\mathrm{K}^{+}$at the stable hydration states $(\mathrm{CWT}=5$, 6 ) are smaller than those for $\mathrm{Na}^{+}(\mathrm{CWT}=5)$, indicating a weaker affinity for water molecules of $\mathrm{K}^{+}$. At low binding, i.e. for CLP $=1,2,3$, the corresponding minimum free-energies are located at hydration levels CWT $=5,4,3$, respectively, resulting in a total coordination number of 6 , which is the same as the experimental hydration number of $\mathrm{K}^{+}[53,54]$. Since the free-energy barriers for the lowest energy bound states of $\mathrm{K}^{+}$are smaller than those for $\mathrm{Na}^{+}$, we should note that its release from lipid binding sites is easier than for sodium.

In the case of calcium, being hydrated in aqueous solution $(\mathrm{CLP}=0)$ is a state about $\sim 16$ $\mathrm{kcal} / \mathrm{mol}$ higher than the global minimum, as shown in Figure 4c. This shows that staying in the 
aqueous solution is greatly unfavorable for $\mathrm{Ca}^{2+}$ compared to being bound to the membranes. In aqueous solution, the most stable hydration states for $\mathrm{Ca}^{2+}$ are $\mathrm{CWT}=6,7$, consistent with experimental hydration number[55, 56]. The most stable bound state for $\mathrm{Ca}^{2+}$ is at $[2,4]$, whereas the total coordination number is 6 . Other stable bound states are at CLP $=3,4,5$ with total coordination number of $4 \sim 6$, which are $2 \sim 4 \mathrm{kcal} / \mathrm{mol}$ higher than the global minimum (see Table 2). These bound states are also the global minima of the FES, which are $12 \sim 16 \mathrm{kcal} / \mathrm{mol}$ lower than the lowest energy unbound states at CLP $=0$. For $\mathrm{Ca}^{2+}$, we obtain a significantly stronger binding to phospholipid membranes over aqueous solution than for $\mathrm{Na}^{+}$and $\mathrm{K}^{+}$where the binding is weak, in agreement with experiment[6]. For the ions considered, the ion binding free-energy has its minimum at the same total coordination number (CLP+CWT), irrespective of CLP or CWT, as shown in Table 2, and coincides with the hydration number in solution. This is a clear indication of the competition between ion binding to water and lipids, and is responsible for the correlation between ion hydration and ion binding specificity at the membranes. Finally, Figure $4 \mathrm{~d}$ shows that in aqueous solution being solvated by 6 water molecules is the most stable state for $\mathrm{Mg}^{2+}$, in accordance with experiments[55, 56]. However, in contrast to the other cations which in solution have several hydration free-energy basins, $\mathrm{Mg}^{2+}$ has only one basin at CWT $=$ 6 , and the corresponding hydration free-energy barrier is very high $(\sim 5 \mathrm{kcal} / \mathrm{mol})$ compared with $\mathrm{Na}^{+}, \mathrm{K}^{+}$and $\mathrm{Ca}^{2+}$. This is due to the strong binding of water molecules to $\mathrm{Mg}^{2+}$, which indicates that magnesium will normally move together with its first water solvation shell, making the change of its most stable hydration state to other hydration states $(\mathrm{CWT}<6)$ and bound states (CLP $>0)$ a rare event phenomenon.

\subsection{Part II: Cholesterol-rich}

The FES of $\mathrm{Na}^{+}$bound to DMPC-cholesterol membranes with cholesterol concentrations [CHOL] $=0-50 \%$ are shown in Figure $5 . \mathrm{Na}^{+}$is considered bound to the membrane for CLP $>0$ and unbound for CLP $=0$. There are a number of bound states (CLP $>0$ ) and several unbound states $(\mathrm{CLP}=0)$ in each FES, and each state can be indexed by (CWT, CLP) as in section 3.1. A common feature for all the [CHOL] cases is the global minimum of the FES located at the $[5,0]$ state, revealing that the hydration with 5 water molecules in the aqueous solution is most favorable for $\mathrm{Na}^{+}$. In addition, we observe a staircase pattern in all of the FES, which is the consequence of the ionic binding competition between water and lipids. As cholesterol concentration increases, the stable bound states (represented by red color in the FES), which are $\sim 1-4 \mathrm{kcal} / \mathrm{mol}$ higher than the global minimum, shift from the region with $\operatorname{CLP} \in\{1,5\}$ and $\mathrm{CWT} \in\{0,5\}$ for $[\mathrm{CHOL}]=0$ $10 \%$ to the region with $\mathrm{CLP} \in\{1,4\}$ and $\mathrm{CWT} \in\{1,5\}$ for $[\mathrm{CHOL}]=20-30 \%$, and further to the region with $\mathrm{CLP} \in\{1,3\}$ and $\mathrm{CWT} \in\{2,5\}$ for $[\mathrm{CHOL}]=40-50 \%$. This visible shifts of stable bound states clearly reveal that the binding competitiveness of lipid head groups has been diminished by cholesterol contents, and that the affinity of $\mathrm{Na}^{+}$to DMPC membranes becomes less favorable as cholesterol concentration increases, which is in agreement with experiments[15].

A more quantitative representation of the above results can be extracted from Figure 6, where the relative free energy $\Delta F$ as a function of CWT for several integer lipid binding levels (CLP) is extracted from the FES of Figure 5. Here we only represent the two extreme cases of [CHOL] $=$ $0 \%$ (continuous lines) and $[\mathrm{CHOL}]=50 \%$ (dashed lines). For CLP $=0$, the hydration free energies of $\mathrm{Na}^{+}$unbound to the membrane are exactly the same for both [CHOL] cases, indicating that, as expected, the hydration of $\mathrm{Na}^{+}$in the aqueous solution is not affected by the content of the membrane. There are several hydration free energy basins, and the most stable one is coordinated with five water molecules (which we establish as the reference state), in agreement with the $\mathrm{Na}^{+}$ hydration number measured by experiments[53,54]. When bound to the membrane (CLP>0), the curves for the two cases become different. For each given CLP, the free energy profiles for [CHOL] $=50 \%$ case increase $1 \sim 2 \mathrm{kcal} / \mathrm{mol}$ compared to the cholesterol-free case $([\mathrm{CHOL}]=0 \%)$. Such 
increase evidences the decrease of $\mathrm{Na}^{+}$affinity to the membrane with high cholesterol content. However, for both [CHOL] cases, the corresponding free energy minima for CLP $=1,2,3,4$, are located at $\mathrm{CWT}=4,3,2,1$, respectively, keeping 5 the total coordination number, which is the same as experimental hydration number of $\mathrm{Na}^{+}$in aqueous solution $[53,54]$.

In Figure 7 , we represent the dependence of the relative binding free energy $\Delta F$ on CLP for membranes with various cholesterol concentrations after integrating out CWT according to

$$
\Delta F\left(s_{1}\right)=-k_{\mathrm{B}} T \log \int \exp ^{-\frac{\Delta F\left(s_{1}, s_{2}\right)}{k_{\mathrm{B}} T}} d s_{2},
$$

where $s_{1}$ and $s_{2}$ are $\mathrm{CVs}, k_{\mathrm{B}}$ is the Boltzmann constant, and $\mathrm{T}$ is the absolute temperature. As cholesterol concentration increases, the free energy profiles corresponding to bound states (CLP $>0$ ) raise monotonically. To understand these changes, we should remember the well-known condensing effect of cholesterol on lipid bilayers, which produces higher membrane rigidity and ordering[57]. See for instance the typical configuration of a $50 \%$ cholesterol rich membrane in Figure 8 . For $[\mathrm{CHOL}] \leq 20 \%$, there are overlaps of the free energy profiles at low binding (CLP $\leq 3)$, indicating that $\mathrm{Na}^{+}$is easily bound to the membranes with low cholesterol concentration. When [CHOL] $\geq 30 \%$, the ionic binding is significantly reduced (free energy increases). Such transition in the ionic binding behavior at $[\mathrm{CHOL}] \approx 30 \%$ coincides with the phase transition point of DMPCcholesterol membranes, in which membranes change from a liquid-disordered phase to a liquidordered phase[13].

We monitor the convergence of the FES by calculating $\Delta \mathrm{F}^{\prime}$, the free energy difference between unbound states $($ CLP $<0.5)$ and bound states $($ CLP $>0.5)$ from the free energy profiles of CLP as the simulations proceed. The average values and standard deviations of $\Delta \mathrm{F}^{\prime}$ calculated from the last 100 ns of well-tempered metadynamics simulations are shown in Figure 9. The monotonic trend indicates that the energy gap between unbound and bound states increases with the increasing cholesterol concentration, which reduces the affinity of $\mathrm{Na}^{+}$to cholesterol-containing membranes. This is in good agreement with the results obtained from the previous Figures 5,6 , and 7 .

The above 2D FES (Figure 6) and 1D free energy $\Delta F$ as a function of CLP (Figure 9) are based on the consideration that all the oxygen atoms from DMPC lipids (i.e. phosphate and carbonyl groups) and cholesterol (hydroxyl group in the polar head) are equivalent binding sites for $\mathrm{Na}^{+}$. In order to understand the contributions of different head groups separately, we calculate the relative free energy $\Delta F$ as a function of CLP between $\mathrm{Na}^{+}$and oxygen atoms from different head groups by applying a reweighting technique[58]. As shown in Figure 10, for a given CLP, the binding free energy follows the order of $\mathrm{C}=\mathrm{O}<\mathrm{PO}_{4}^{-}<-\mathrm{OH}$. Therefore, $\mathrm{C}=\mathrm{O}$ is the most favorable binding site for $\mathrm{Na}^{+}$, followed by $\mathrm{PO}_{4}^{-}$and then the $-\mathrm{OH}$ group of cholesterol. For DMPC binding sites $(\mathrm{C}=\mathrm{O}$ and $\mathrm{PO}_{4}^{-}$), we observe higher binding free energy basins and higher binding free energy barriers for higher cholesterol concentration, which is in accordance with above results obtained when different CLP are considered equivalent. The situation for the CLP between $\mathrm{Na}^{+}$and cholesterol is radically different. At low cholesterol concentration (10\%), only one cholesterol oxygen can be attached to $\mathrm{Na}^{+}$. For $[\mathrm{CHOL}] \geq 20 \%$, it would be possible for $\mathrm{Na}^{+}$to bind up to two cholesterol oxygens. However, the free energy barriers for high cholesterol concentrations $(40-50 \%)$ are lower than those for the medium ones $(20-30 \%)$, which is in contrast to the trend observed for DMPC binding sites where higher free energy barrier corresponds to higher cholesterol concentration.

\section{Conclusions}

In this study we have provided a quantitative characterization of the coordination numbers of biologically relevant metal cations $\left(\mathrm{Na}^{+}, \mathrm{K}^{+}, \mathrm{Ca}^{2+}\right.$, and $\left.\mathrm{Mg}^{2+}\right)$ at DMPC phospholipid membranes 
with no cholesterol contents. With the help of well-tempered metadynamics simulations we have calculated the free-energies of the binding states, defined by the ion coordination to lipid (CLP) and water (CWT) oxygen atoms. The results from metadynamics simulations have been obtained using previously equilibrated samples obtained from MD simulations performed with the recently parameterized CHARMM36[41] force field, which strongly influenced the quality of the physical information reported here. Our results indicate that $\mathrm{Na}^{+}$and $\mathrm{K}^{+}$are more likely to stay in the aqueous solution, and can easily bind to $1 \sim 3$ lipid oxygens by overcoming free-energy barriers of $1 \sim 2 \mathrm{kcal} / \mathrm{mol}$. $\mathrm{Ca}^{2+}$ is most stable when bound to 4 lipid oxygens of the membranes, and the corresponding bound basin is $\sim 16 \mathrm{kcal} / \mathrm{mol}$ lower than the unbound states in the aqueous solution. $\mathrm{Mg}^{2+}$ has a strong affinity to hydration water, which makes the direct binding to the membranes difficult. When bound to the membranes, the cations' most favorable total coordination numbers with water and lipid oxygens are 5, 6, and 6 for $\mathrm{Na}^{+}, \mathrm{K}^{+}$, and $\mathrm{Ca}^{2+}$, respectively. Such coordination numbers coincide with their corresponding hydration numbers in bulk, suggesting a competition between ion binding to water and lipids.

In the second part of the paper, we obtained FES for $\mathrm{Na}^{+}$bound to DMPC-cholesterol membranes with several concentrations of cholesterol by means of well-tempered metadynamics simulations with the same two collective variables as in the previous study (CLP, CWT). The free energy surfaces reveal the competition between binding of ion to water and to lipids. However, the binding competitiveness of lipid head groups is diminished by cholesterol contents. As cholesterol concentration increases, the ionic affinity to the membrane decreases, which is in agreement with experiments[15]. When cholesterol concentration [CHOL] $\geq 30 \%$, the ionic binding is significantly reduced. Such transition in the ionic binding behavior at [CHOL] $\approx 30 \%$ coincides with the phase transition point of DMPC-cholesterol membranes, in which membranes change from a liquid-disordered phase to a liquid-ordered phase. In contrast, the hydration free energies of $\mathrm{Na}^{+}$ in aqueous solution are not affected by the cholesterol content of membranes. The most stable hydration for $\mathrm{Na}^{+}$with five water molecules is in good agreement with experiments. We have also evaluated the contributions of different lipid head groups to the binding free energy separately. The DMPC's carbonyl group $(\mathrm{C}=\mathrm{O})$ is the most favorable binding site for $\mathrm{Na}^{+}$, followed by DMPC's phosphate group $\left(\mathrm{PO}_{4}^{-}\right)$and then the hydroxyl group $(-\mathrm{OH})$ of cholesterol.

It should be pointed out that due to the high computational demands of the $2 \mathrm{D}$ well-tempered metadynamics simulations, we used a small membrane, containing 72 DMPC/cholesterol molecules. Although our tests using unbiased simulations of systems with 72 and $128 \mathrm{DMPC} /$ cholesterol molecules suggest that large size effects can be discarded in describing ion binding to the membrane, a possible contribution from the finite size of the system cannot be completely ignored. The effect of the limited size of the system can also be relevant in regards to the heterogeneity of the membrane, which is important to ensure a proper sampling of the DMPC/cholesterol configurations of the membrane. We have run long enough simulations to allow complete mixing of lipids, but there might be size effects on the mixing of DMPC and cholesterol molecules which our approach was unable to evaluate. The method employed can be widely applied to explore the free energy landscapes of ions at complex biological interfaces. Furthermore, provided the importance in a variety of biological processes of the interaction of ions and charged interfaces in aqueous solution, our approach could be extended to explore other problems in colloidal chemistry and biology, and could be helpful to deepen our understanding of specific ion effects on soft matter and biological systems. However, we should stress again the importance of the choice of the force field employed, since the description of the correct behavior of ions like calcium and magnesium is still elusive for most of the available potential models[25]. 


\section{Acknowledgement}

The author gratefully acknowledges financial support from Spanish Ministry of Economy and Knowledge (grant FIS2015-66879-C2-1-P) and computer resources, technical expertise and assistance provided by the Red Española de Supercomputación through Barcelona Supercomputing Centre (project FI-2015-1-0007). 
Tables

Table 1. Simulation parameters for cholesterol-free simulations

\begin{tabular}{l|l|l|l|l}
\hline Parameter & $\mathrm{Na}^{+}$ & $\mathrm{K}^{+}$ & $\mathrm{Ca}^{2+}$ & $\mathrm{Mg}^{2+}$ \\
\hline$d_{0}$ of CLP $[\AA]$ & 2.3 & 2.64 & 2.12 & 1.85 \\
$r_{0}$ of CLP $[\AA]$ & 0.25 & 0.32 & 0.12 & 0.13 \\
$d_{0}$ of CWT $[\AA]$ & 2.35 & 2.7 & 2.24 & 1.95 \\
$r_{0}$ of CWT $[\AA]$ & 0.25 & 0.36 & 0.22 & 0.18 \\
Gaussian width of CLP & 0.2 & 0.2 & 0.2 & 0.2 \\
Gaussian width of CWT & 0.2 & 0.2 & 0.2 & 0.2 \\
Starting hill [kcal/mol] & 0.3 & 0.3 & 0.5 & 0.5 \\
Deposition stride [ps] & 1 & 1 & 1 & 1 \\
Bias factor & 5 & 3 & 20 & 15 \\
Simulation time $[\mathrm{ns}]$ & 800 & 800 & 800 & 100 \\
\hline
\end{tabular}

Table 2. Free-energies for the lowest states of $\mathrm{Na}^{+}, \mathrm{K}^{+}$, and $\mathrm{Ca}^{2+}$ with respect to the global minimum.

\begin{tabular}{l|l|l|l|l}
\hline \multirow{2}{*}{ Ion } & \multicolumn{3}{|c|}{ binding state } & F $[\mathrm{kcal} / \mathrm{mol}]$ \\
\cline { 2 - 4 } & CLP & CWT & CLP+CWT & \\
\hline $\mathrm{Na}^{+}$ & 0 & 5 & 5 & 0.0 \\
& 1 & 4 & 5 & 1.28 \\
& 2 & 3 & 5 & 1.69 \\
& 3 & 2 & 5 & 2.69 \\
\hline $\mathrm{K}^{+}$ & 0 & 6 & 6 & 0.0 \\
& 0 & 5 & 5 & 0.11 \\
& 1 & 5 & 6 & 1.23 \\
& 2 & 4 & 6 & 1.46 \\
& 3 & 3 & 6 & 1.78 \\
& 4 & 2 & 6 & 2.26 \\
\hline $\mathrm{Ca}^{2+}$ & 4 & 2 & 6 & 0.0 \\
& 4 & 0 & 4 & 2.07 \\
& 5 & 0 & 5 & 2.74 \\
& 3 & 3 & 6 & 3.13 \\
\hline
\end{tabular}

Simulation time [ns] 


\section{Figures}
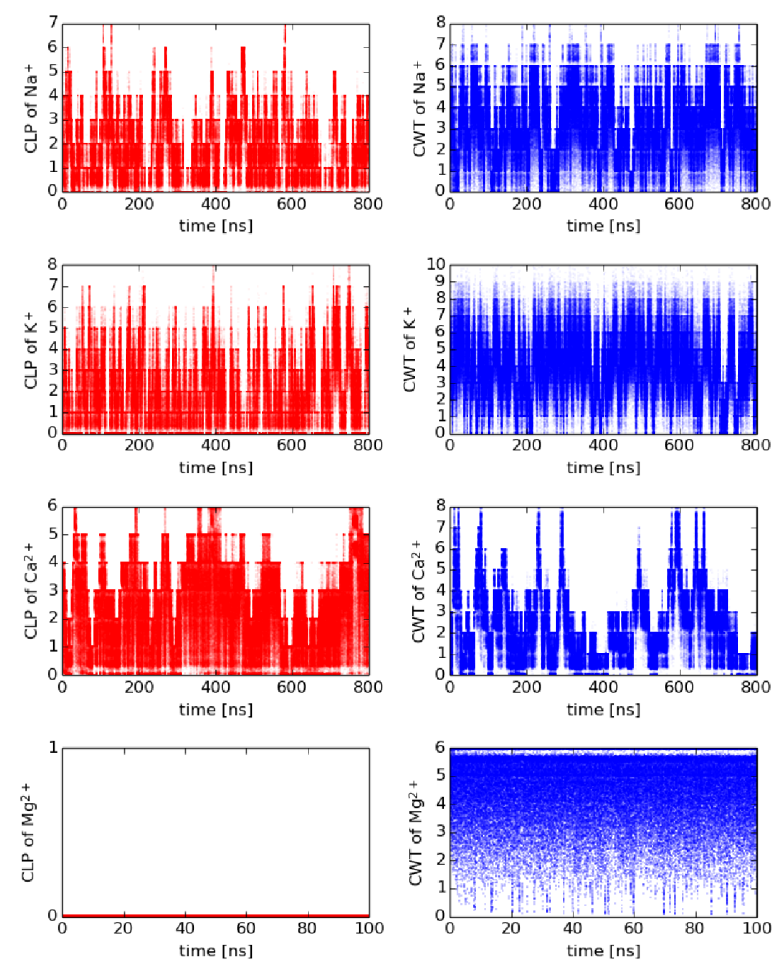

Figure 1. Well-tempered metadynamics trajectories of $\mathrm{Na}^{+}, \mathrm{K}^{+}, \mathrm{Ca}^{2+}$, and $\mathrm{Mg}^{2+}$. Left panels, red traces: CLP as a function of time. Right panels, blue traces: CWT as a function of time. The traces of $\mathrm{Mg}^{2+}$ show that CLP $=0$ during $100 \mathrm{~ns}$, indicating that the bound states of $\mathrm{Mg}^{2+}$ to lipid oxygens (CLP $\geq 1$ ) are separated from the unbound states in the aqueous solution (CLP $=0$ ) by high free-energy barriers, and could not be appropriately sampled by our calculations. 

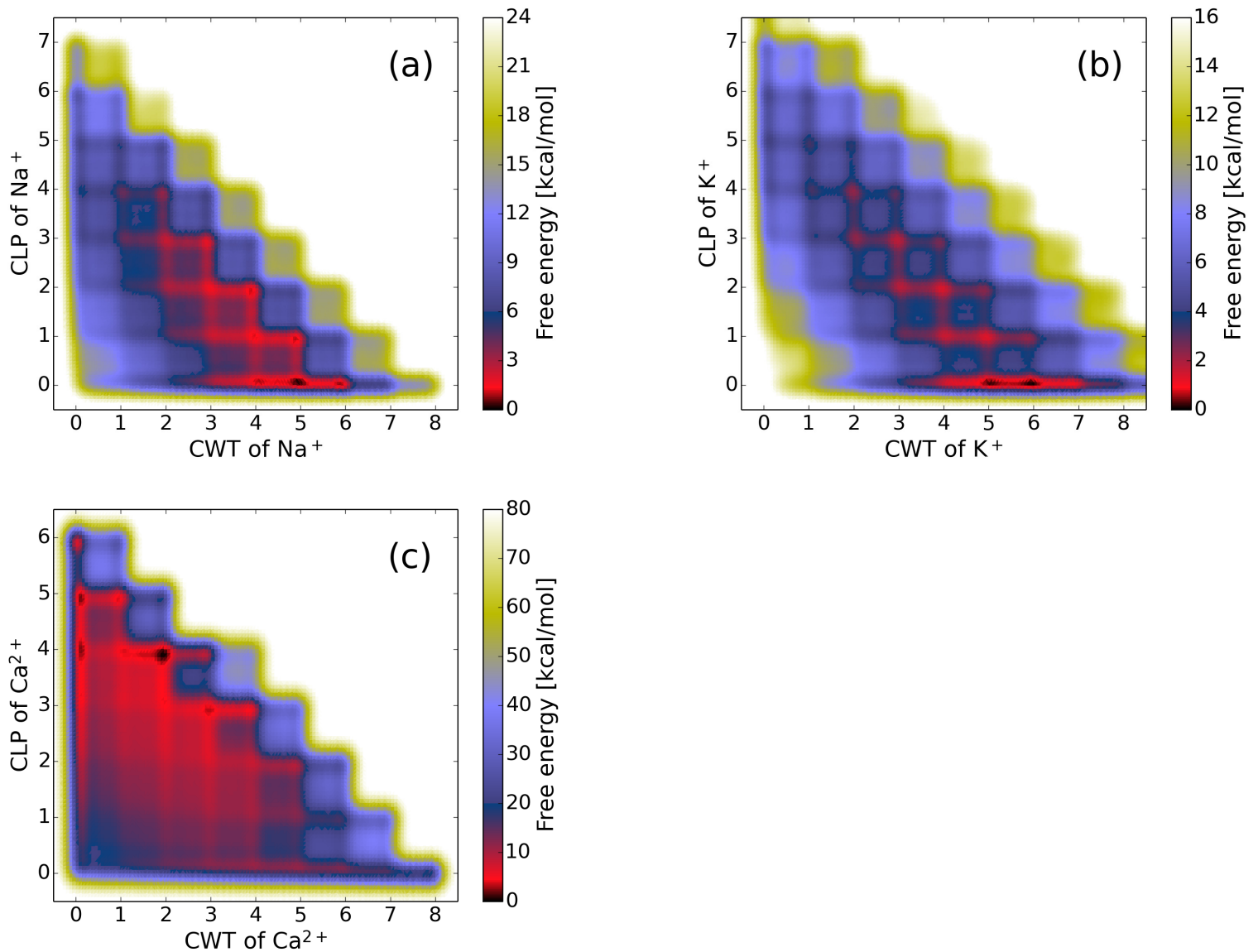

Figure 2. Free-energy surface as a function of the coordination number of water oxygens (CWT) and the coordination number of lipid oxygens (CLP) for (a) $\mathrm{Na}^{+},(\mathrm{b}) \mathrm{K}^{+}$and (c) $\mathrm{Ca}^{2+}$ at cholesterol-free DMPC membrane surfaces.

(a)

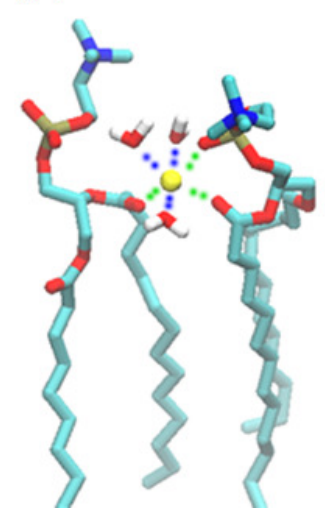

(b)

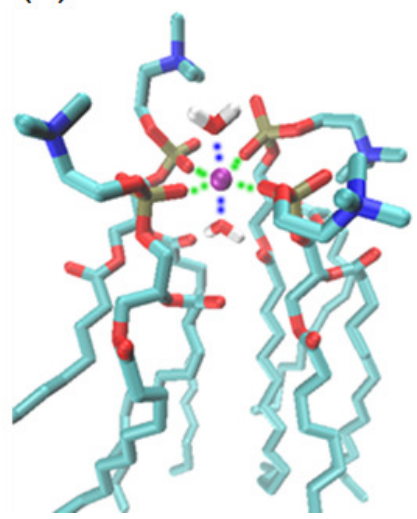

Figure 3. (a) A representative bound state for $\mathrm{Na}^{+}$coordinated to 3 lipid oxygens and 3 water molecules. (b) The most stable bound state for $\mathrm{Ca}^{2+}$ coordinated to 4 lipid oxygens and 2 water molecules. $\mathrm{Na}^{+}$is in yellow and $\mathrm{Ca}^{2+}$ is in purple. The binding to lipid oxygens and to water oxygens are shown in green and blue respectively. 

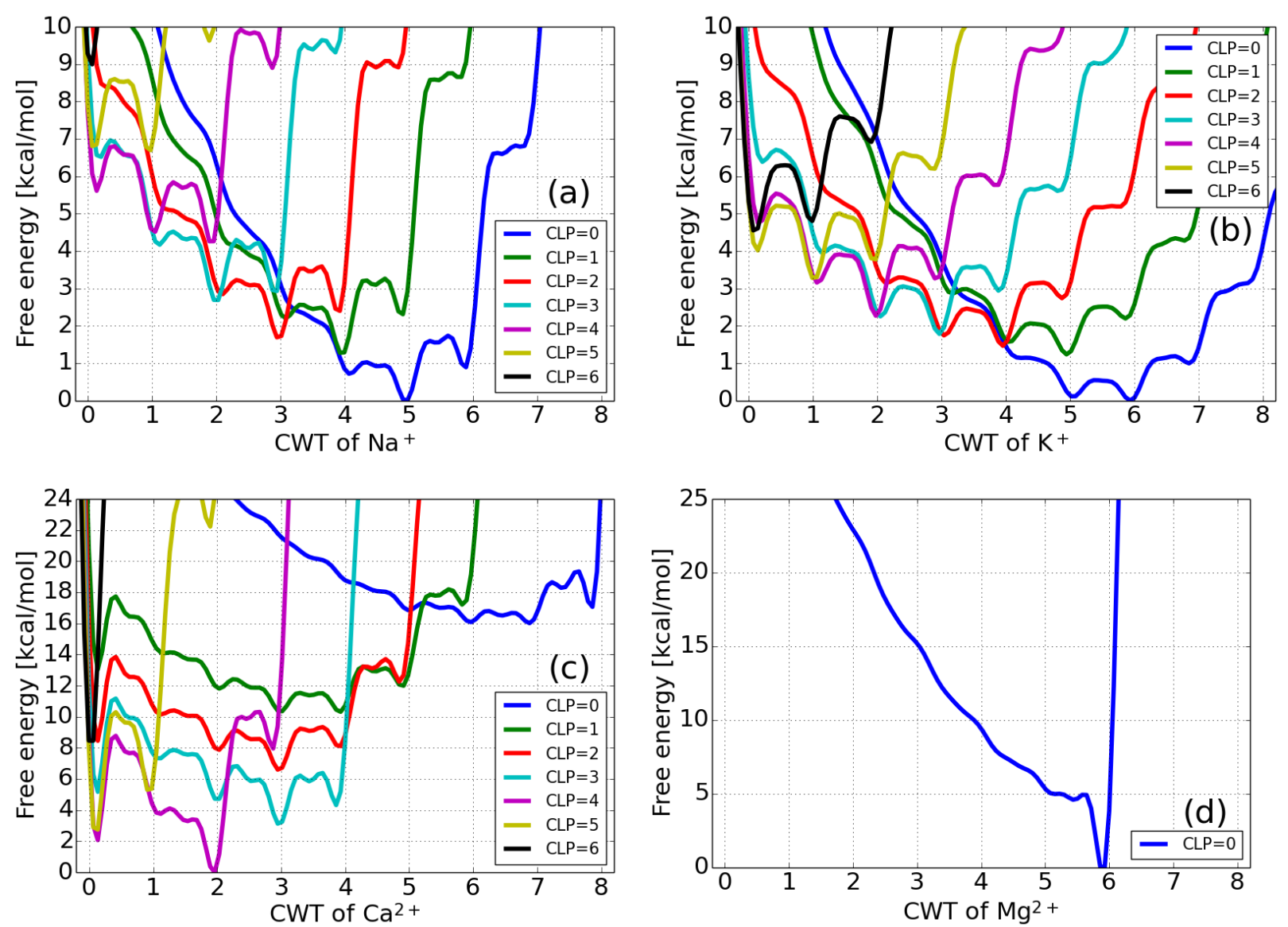

Figure 4. Free-energy as a function of CWT at various CLP for $\mathrm{Na}^{+}(\mathrm{a}), \mathrm{K}^{+}$(b), $\mathrm{Ca}^{2+}$ (c) and $\mathrm{Mg}^{2+}$ (d). The data for $\mathrm{Na}^{+}$, $\mathrm{K}^{+}$, and $\mathrm{Ca}^{2+}$ are extracted from Figure 1 . For $\mathrm{Mg}^{2+}$, only the case with CLP $=0$ is considered. 

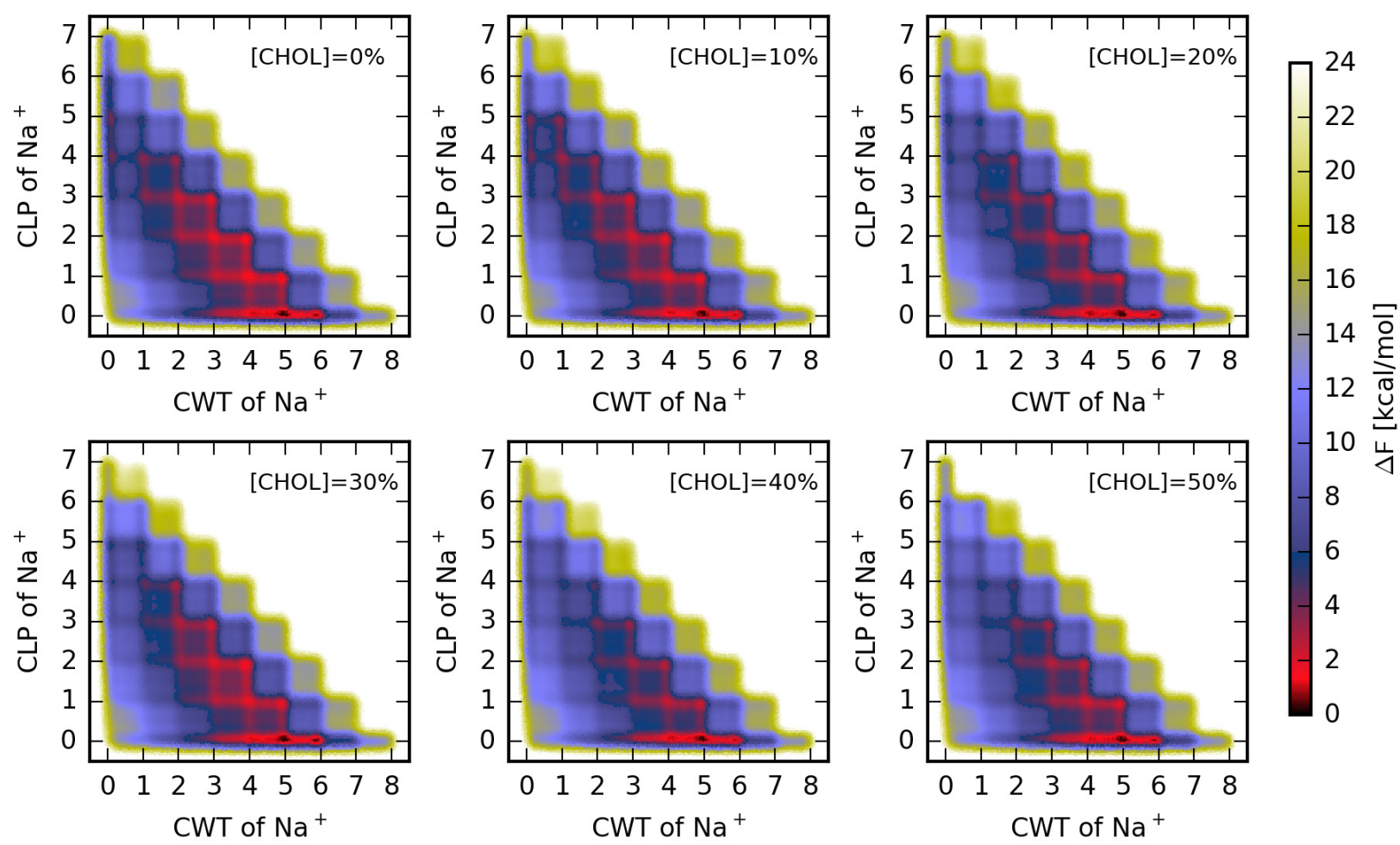

Figure 5. Binding free energy difference $\Delta \mathrm{F}$ as a function of the coordination number of lipid oxygens (CLP) and the coordination number of water oxygens (CWT) for $\mathrm{Na}^{+}$bound to DMPC membranes with cholesterol concentrations [CHOL] varying between $0 \%$ and $50 \%$. The origin of free energy is given by the binding state with minimum free energy, with CLP=0 and $\mathrm{CWT}=5$. 


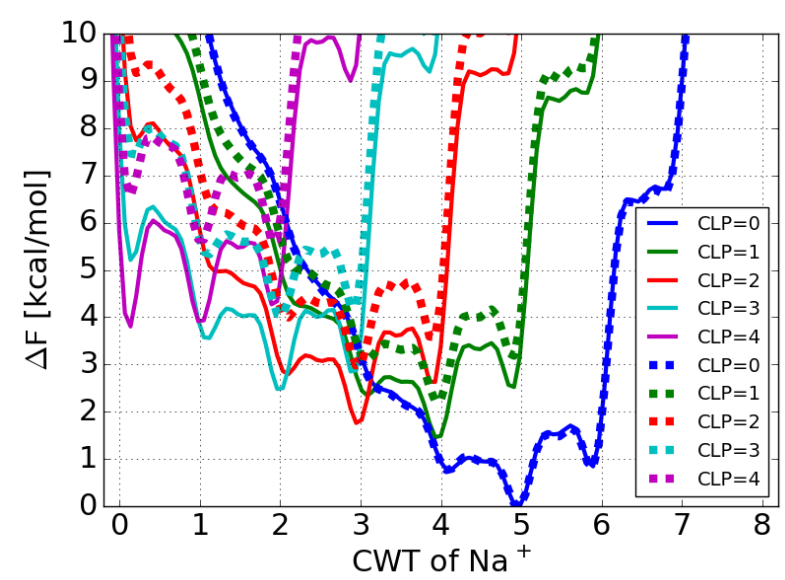

Figure 6. Relative free energy $\Delta \mathrm{F}$ as a function of CWT at CLP $=0-4$ for $\mathrm{Na}^{+}$bound to DMPC membranes with cholesterol concentration [CHOL] of $0 \%$ (continuous lines) and 50\% (dashed lines). The data are extracted from Figure 4.

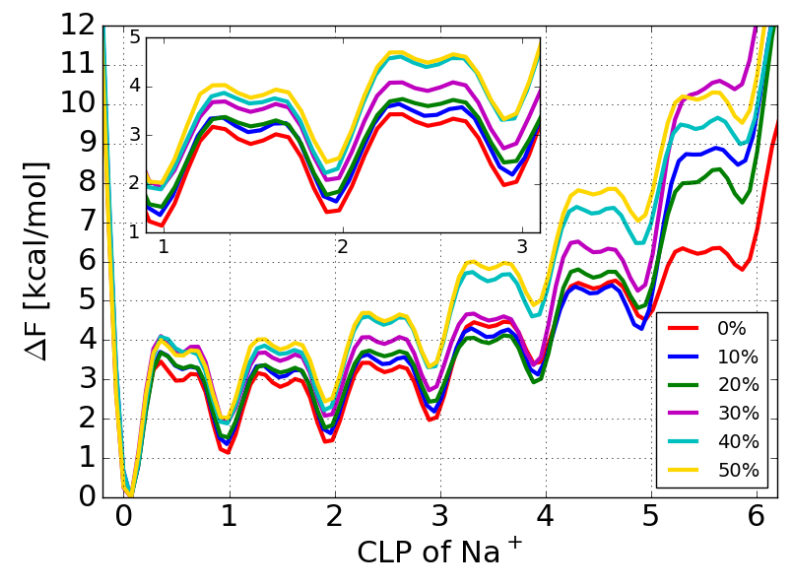

Figure 7. Relative free energy $\Delta \mathrm{F}$ as a function of CLP for $\mathrm{Na}^{+}$bound to membranes with cholesterol concentration [CHOL] varying between $0 \%$ and $50 \%$. (Inset) Zoom in the low CLP region.

\section{References}

[1] Lo Nostro P, Ninham BW. Hofmeister Phenomena: an Update on Ion Specificity in Biology. Chem Rev. 2012;112:2286-2322.

[2] Kunz W. Specific Ion Effects in Colloidal and Biological Systems. Curr Opin Colloid Interface Sci. 2010; 15:34-39.

[3] Berkowitz ML, Bostick DL, Pandit S. Aqueous Solutions Next to Phospholipid Membrane Surfaces: Insights from Simulations. Chem Rev. 2006;106:1527-1539.

[4] Berkowitz ML, Vácha R. Aqueous Solutions at the Interface with Phospholipid Bilayers. Acc Chem Res. 2012;45:74-82.

[5] Binder H, Zschörnig O. The Effect of Metal Cations on the Phase Behavior and Hydration Characteristics of Phospholipid Membranes. Chem Phys Lipids. 2002;115:39-61.

[6] Casillas-Ituarte NN, Chen X, Castada H, et al. Na+ and Ca2+ Effect on the Hydration and Orientation of the Phosphate Group of DPPC at Air-Water and Air-Hydrated Silica Interfaces. J Phys Chem B. 2010;114:9485-9495.

[7] Jurkiewicz P, Cwiklik L, Vojtíśková A, et al. Structure, Dynamics, and Hydration of POPC/POPS Bilayers Suspended in $\mathrm{NaCl}, \mathrm{KCl}$, and $\mathrm{CsCl}$ Solutions. Biochim Biophys Acta - Biomembr. 2012;1818:609616.

[8] Klasczyk B, Knecht V, Lipowsky R, et al. Interactions of Alkali Metal Chlorides with Phosphatidyl- 


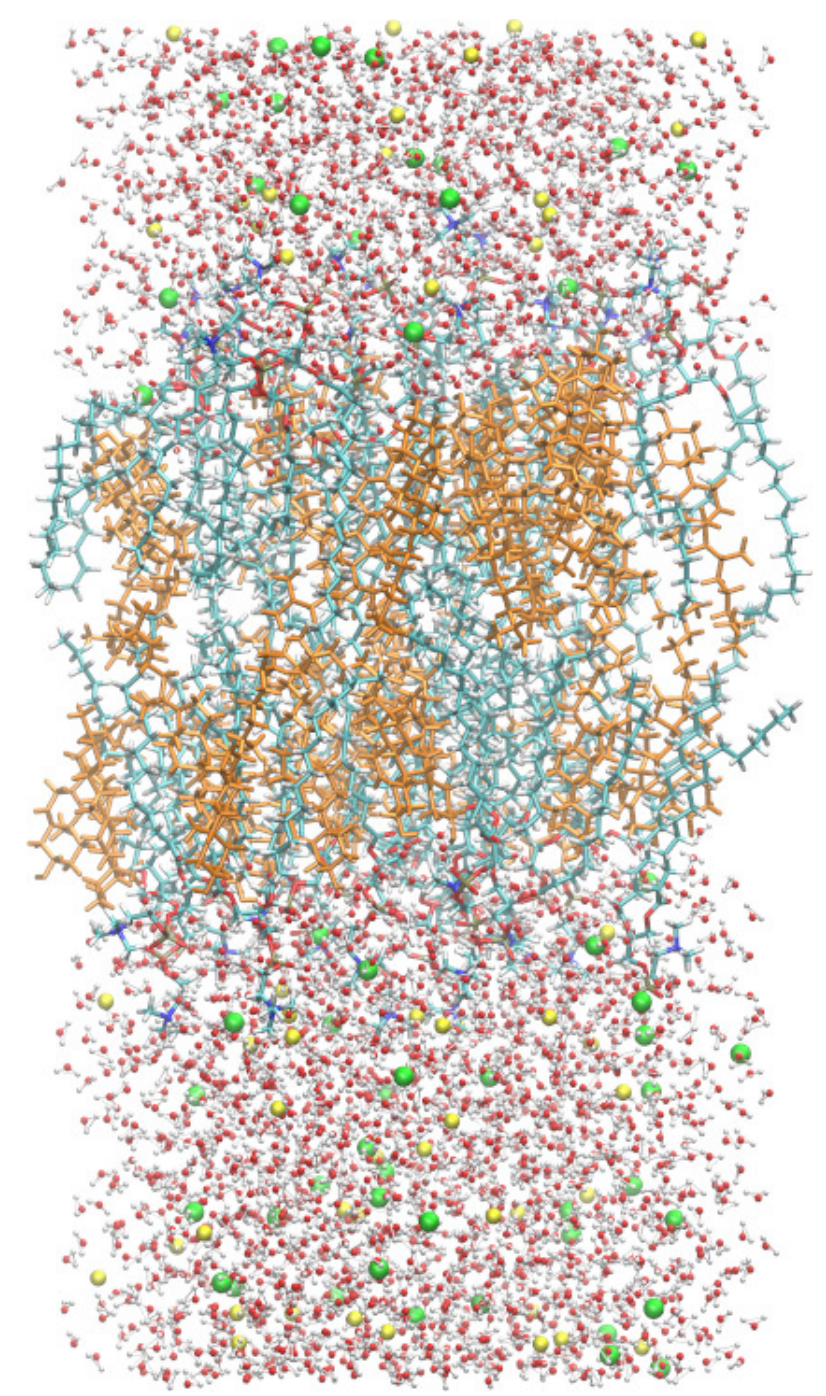

Figure 8. Snapshot of a typical DMPC-cholesterol NaCl membrane, with 505 of cholesterol contents. $\mathrm{Na}^{+}$(green), $\mathrm{Cl}^{-}$(yellow), cholesterol (orange), water's oxygen (red), water's hydrogen (white), DPMC (skeleton, blue).

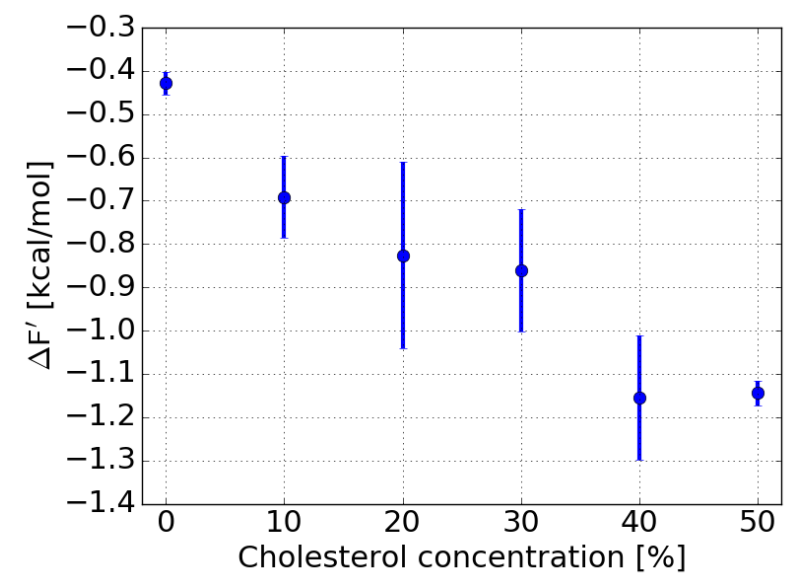

Figure 9. Average values and standard deviations of $\Delta \mathrm{F}^{\prime}$, the free energy difference between unbound states $(\mathrm{CLP}<0.5)$ and

$$
\text { bound states (CLP > 0.5) for different cholesterol concentrations. }
$$

(a) (1)

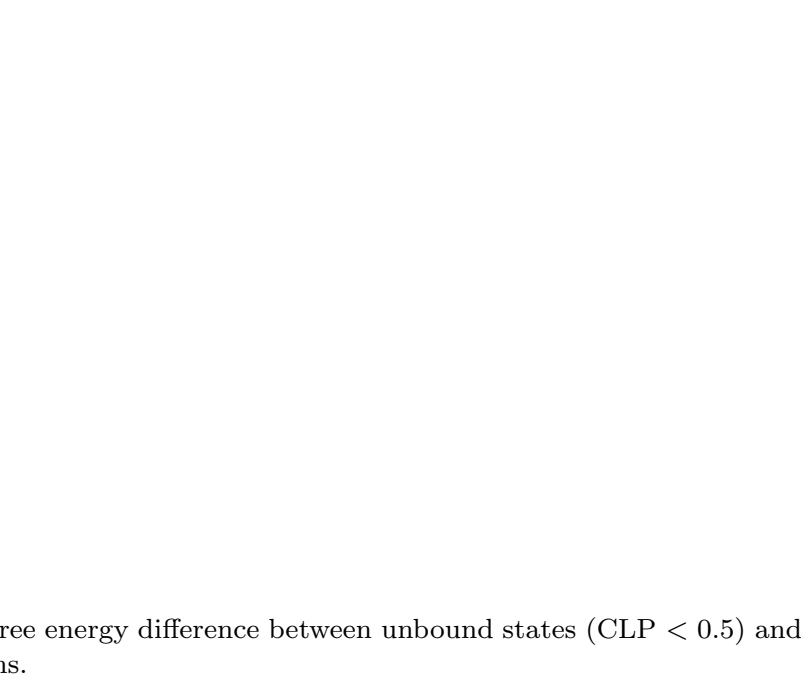

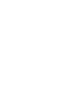

. 

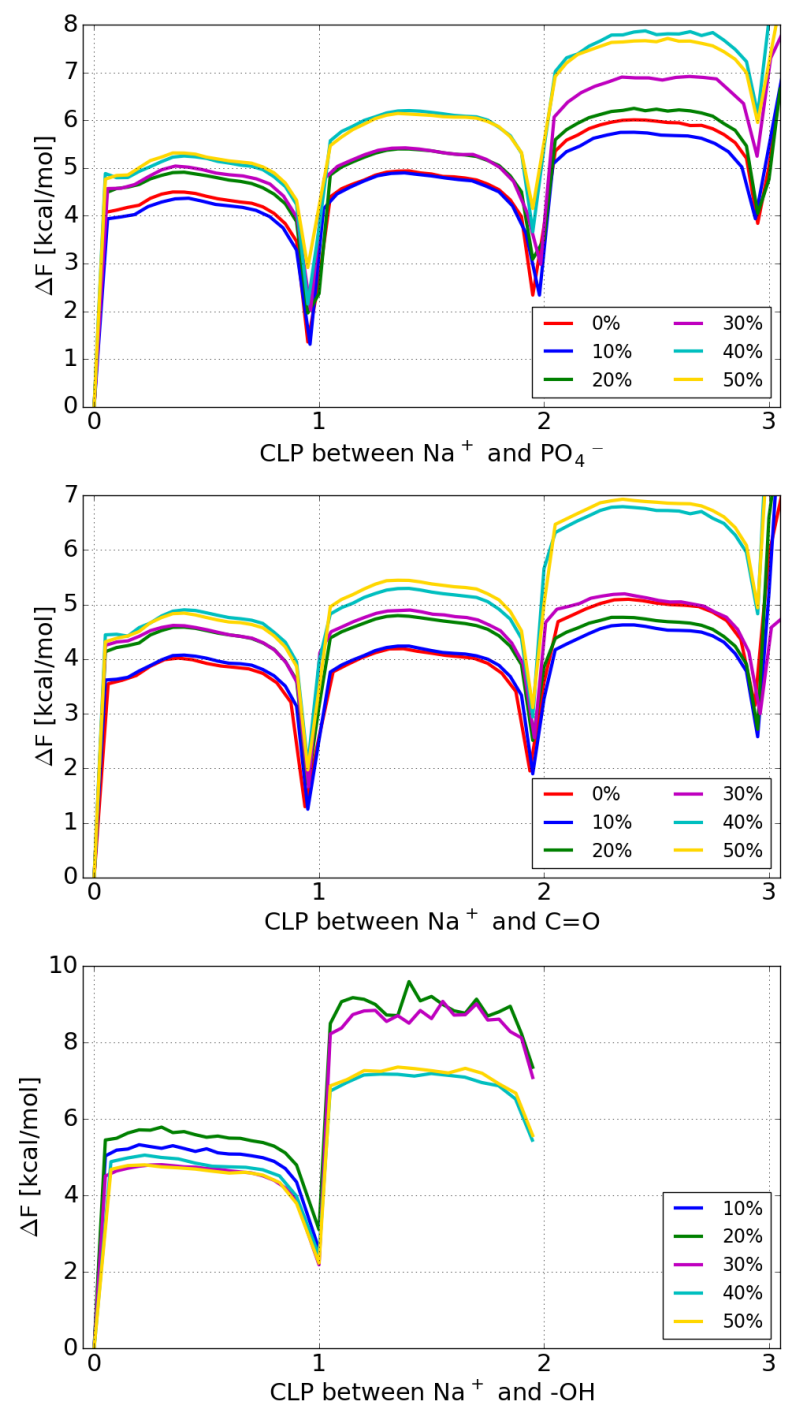

Figure 10. Relative free energy $\Delta \mathrm{F}$ as a function of CLP between $\mathrm{Na}^{+}$and oxygen atoms from different lipid head groups with cholesterol concentration $[\mathrm{CHOL}]$ varying between $0 \%$ and $50 \%$. The three different lipid head groups are: DMPC's phosphate group $\mathrm{PO}_{4}^{-}$(top figure); DMPC's carbonyl group $\mathrm{C}=\mathrm{O}$ (middle figure); and cholesterol's hydroxyl group -OH (bottom figure).

choline Vesicles. Langmuir. 2010;26:18951-18958.

[9] Pandit SA, Bostick D, Berkowitz ML. Molecular Dynamics Simulation of a Dipalmitoylphosphatidylcholine Bilayer with NaCl. Biophys J. 2003;84:3743-3750.

[10] Vist MR, Davis JH. Phase Equilibria of Cholesterol / Dipalmitoylphosphatidylcholine Mixtures: 2H Nuclear Magnetic Resonance and Differential Scanning Calorimetry. Biochemistry. 1990;29:451-464.

[11] Berkowitz ML. Detailed Molecular Dynamics Simulations of Model Biological Membranes Containing Cholesterol. Biochim Biophys Acta. 2009;1788:86-96.

[12] de Meyer FJM, Benjamini A, Rodgers JM, et al. Molecular Simulation of the DMPC-Cholesterol Phase Diagram. J Phys Chem B. 2010;114:10451-10461.

[13] Armstrong CL, Barrett MA, Hiess A, et al. Effect of Cholesterol on the Lateral Nanoscale Dynamics of Fluid Membranes. Eur Biophys J. 2012;41:901-913.

[14] Iraolagoitia XLR, Martini MF. Ca(2+) Adsorption to Lipid Membranes and the Effect of Cholesterol in Their Composition. Colloids Surf, B. 2010;76:215-220.

[15] Magarkar A, Dhawan V, Kallinteri P, et al. Cholesterol Level Affects Surface Charge of Lipid Membranes in Saline Solution. Sci Rep. 2014;4:5005.

[16] Böckmann RA, Hac A, Heimburg T, et al. Effect of Sodium Chloride on a Lipid Bilayer. Biophys J. 
2003;85:1647-1655.

[17] Gurtovenko AA. Asymmetry of Lipid Bilayers Induced by Monovalent Salt: Atomistic MolecularDynamics Study. J Chem Phys. 2005;122:244902.

[18] Cordomí A, Edholm O, Perez JJ. Effect of Ions on a Dipalmitoyl Phosphatidylcholine Bilayer. A Molecular Dynamics Simulation Study. J Phys Chem B. 2008;112:1397-1408.

[19] Vácha R, Siu SWI, Petrov M, et al. Effects of Alkali Cations and Halide Anions on the DOPC Lipid Membrane. J Phys Chem A. 2009;113:7235-7243.

[20] Barducci A, Bonomi M, Parrinello M. Metadynamics. Wiley Interdiscip Rev Comput Mol Sci. 2011; 1:826-843.

[21] Chipot C. Frontiers in Free-Energy Calculations of Biological Systems. Wiley Interdiscip Rev Comput Mol Sci. 2014;4:71-89.

[22] Hansen N, Van Gunsteren WF. Practical Aspects of Free-energy Calculations: A Review. J Chem Theory Comput. 2014;10:2632-2647.

[23] Barducci A, Bussi G, Parrinello M. Well-Tempered Metadynamics: A Smoothly Converging and Tunable Free-Energy Method. Phys Rev Lett. 2008;100:020603.

[24] Valley C, Perlmutter J, Braun A, et al. Nacl interactions with phosphatidylcholine bilayers do not alter membrane structure but induce long-range ordering of ions and water. J Membrane Biol. 2011; 244:35-42.

[25] Catte A, Girych M, Javanainen M, et al. Molecular electrometer and binding of cations to phospholipid bilayers. Phys Chem Chem Phys. 2016;18:32560-32569; Available from: http://dx.doi.org/10.1039/C6CP04883H.

[26] Jämbeck JPM, Lyubartsev AP. Exploring the Free Energy Landscape of Solutes Embedded in Lipid Bilayers. J Phys Chem Lett. 2013;4:1781-1787.

[27] Wennberg CL, Van Der Spoel D, Hub JS. Large Influence of Cholesterol on Solute Partitioning into Lipid Membranes. J Am Chem Soc. 2012;134:5351-5361.

[28] Khavrutskii IV, Gorfe AA, Lu B, et al. Free Energy for the Permeation of Na+ and Cl- Ions and Their Ion-Pair Through a Zwitterionic Dimyristoyl Phosphatidylcholine Lipid Bilayer by Umbrella Integration with Harmonic Fourier Beads. J Am Chem Soc. 2009;131:1706-1716.

[29] Vorobyov I, Olson TE, Kim JH, et al. Ion-Induced Defect Permeation of Lipid Membranes. Biophys J. 2014;106:586-597.

[30] Herbette L, Napolitano CA, McDaniel RV. Direct Determination of the Calcium Profile Structure for Dipalmitoyllecithin Multilayers Using Neutron Diffraction. Biophys J. 1984;46:677-685.

[31] Böckmann RA, Grubmüller H. Multistep Binding of Divalent Cations to Phospholipid Bilayers: A Molecular Dynamics Study. Angew Chem, Int Ed. 2004;43:1021-1024.

[32] Gurtovenko AA, Vattulainen I. Effect of $\mathrm{NaCl}$ and $\mathrm{KCl}$ on Phosphatidylcholine and Phosphatidylethanolamine Lipid Membranes: Insight from Atomic-Scale Simulations for Understanding SaltInduced Effects in the Plasma Membrane. J Phys Chem B. 2008;112:1953-1962.

[33] Laio A, Parrinello M. Escaping Free-Energy Minima. Proc Natl Acad Sci USA. 2002;99:12562-12566.

[34] Jo S, Kim T, Iyer VG, et al. CHARMM-GUI: A Web-Based Graphical User Interface for CHARMM. J Comput Chem. 2008;29:1859-1865.

[35] Jo S, Lim JB, Klauda JB, et al. CHARMM-GUI Membrane Builder for Mixed Bilayers and Its Application to Yeast Membranes. Biophys J. 2009;97:50-58.

[36] Pasenkiewicz-Gierula M, Takaoka Y, Miyagawa H, et al. Hydrogen Bonding of Water to Phosphatidylcholine in the Membrane as Studied by a Molecular Dynamics Simulation: Location, Geometry, and Lipid-Lipid Bridging via Hydrogen-Bonded Water. J Phys Chem A. 1997;101:3677-3691.

[37] Yang J, Calero C, Martí J. Diffusion and Spectroscopy of Water and Lipids in Fully Hydrated Dimyristoylphosphatidylcholine Bilayer Membranes. J Chem Phys. 2014;140:104901.

[38] Jorgensen WL, Chandrasekhar J, Madura JD, et al. Comparison of Simple Potential Functions for Simulating Liquid Water. J Chem Phys. 1983;79:926-935.

[39] A D MacKerell J, Bashford D, Bellott M, et al. All-atom empirical potential for molecular modeling and dynamics studies of proteins. J Phys Chem B. 1998;102:3586-3616.

[40] Phillips JC, Braun R, Wang W, et al. Scalable Molecular Dynamics with NAMD. J Comput Chem. 2005;26:1781-1802.

[41] Klauda JB, Venable RM, Freites JA, et al. Update of the CHARMM All-Atom Additive Force Field for Lipids: Validation on Six Lipid Types. J Phys Chem B. 2010;114:7830-7843. 
[42] Ryckaert JP, Ciccotti G, Berendsen HJ. Numerical Integration of the Cartesian Equations of Motion of a System with Constraints: Molecular Dynamics of N-Alkanes. J Comput Phys. 1977;23:327-341.

[43] Miyamoto S, Kollman PA. Settle: An Analytical Version of the SHAKE and RATTLE Algorithm for Rigid Water Models. J Comput Chem. 1992;13:952-962.

[44] Essmann U, Perera L, Berkowitz ML, et al. A Smooth Particle Mesh Ewald Method. J Chem Phys. 1995;103:8577.

[45] Hoover W. Canonical Dynamics: Equilibrium Phase-Space Distributions. Phys Rev A. 1985;31:16951697.

[46] Feller SE, Zhang Y, Pastor RW, et al. Constant Pressure Molecular Dynamics Simulation: The Langevin Piston Method. J Chem Phys. 1995;103:4613.

[47] Tribello GA, Bonomi M, Branduardi D, et al. PLUMED 2: New Feathers for an Old Bird. Comput Phys Commun. 2014;185:604-613.

[48] Bonomi M, Branduardi D, Bussi G, et al. PLUMED: A Portable Plugin for Free-energy Calculations with Molecular Dynamics. Comput Phys Commun. 2009;180:1961-1972.

[49] Venable RM, Luo Y, Gawrisch K, et al. Simulations of anionic lipid membranes: Development of interaction-specific ion parameters and validation using nmr data. J Phys Chem B. 2013;117:1018310192.

[50] Fyta M, Netz RR. Ionic force field optimization based on single-ion and ion-pair solvation properties: Going beyond standard mixing rules. J Chem Phys. 2012;136:124103.

[51] Marrink SJ, , Mark AE. Effect of undulations on surface tension in simulated bilayers. J Phys Chem B. 2001;105:6122-6127.

[52] Allnér O, Nilsson L, Villa A. Magnesium Ion-Water Coordination and Exchange in Biomolecular Simulations. J Chem Theory Comput. 2012;8:1493-1502.

[53] Mancinelli R, Botti A, Bruni F, et al. Hydration of Sodium, Potassium, and Chloride Ions in Solution and the Concept of Structure Maker/Breaker. J Phys Chem B. 2007;111:13570-13577.

[54] Varma S, Rempe SB. Coordination Numbers of Alkali Metal Ions in Aqueous Solutions. Biophys Chem. 2006;124:192-199.

[55] Peschke M, Blades AT, Kebarle P. Hydration Energies and Entropies for Mg2+, Ca2+, Sr2+, and Ba2+ from Gas-Phase Ion-Water Molecule Equilibria Determinations. J Phys Chem. 1998;102:9978-9985.

[56] Bush MF, O'Brien JT, Prell JS, et al. Hydration of Alkaline Earth Metal Dications: Effects of Metal Ion Size Determined Using Infrared Action Spectroscopy. J Am Chem Soc. 2009;131:13270-13277.

[57] Hung WC, Lee MT, Chen FY, et al. The Condensing Effect of Cholesterol in Lipid Bilayers. Biophys J. 2007;92:3960-3967.

[58] Bonomi M, Barducci A, Parrinello M. Reconstructing the Equilibrium Boltzmann Distribution from Well-Tempered Metadynamics. J Comput Chem. 2009;30:1615-1621. 